\title{
Apoptotic mitochondria prime anti-tumour immunity
}

\author{
Kate McArthur $\mathbb{D}^{1,2}$ and Benjamin T. Kile ${ }^{1,2}$
}

Immunogenic cell death (ICD) - the ability of a dying cell to activate an immune response-is attracting increasing interest as a biological phenomenon, and as a potential means to augment anti-tumour immunity. In a recent article, Yamazaki and colleagues explore the role of autophagy in limiting the immunogenicity of cell death following radiation therapy and, in-turn, the abscopal effect ${ }^{1}$.

Apoptosis, first described in 1972 by Kerr, Wyllie and Currie $^{2}$, is the best characterised of all programmed cell death modalities. In contrast to its counterparts such as necroptosis and pyroptosis, apoptosis proceeds in an immunologically silent manner. Key to this silence is the function of a family of proteases called Caspases (cysteinerequiring aspartate-specific proteases). Caspases accelerate cellular demolition and clearance. It is increasingly apparent that this activity functions to prevent ICD, both directly (e.g., nuclear fragmentation and the inhibition of protein-synthesis ensures dying cells do not secrete inflammatory cytokines, and the direct deactivation of danger-associated molecular patterns (DAMPs) prevents unwanted immune activation of neighbouring cells) and indirectly (e.g., loss of cellular adhesion, membrane blebbing and display of 'eat-me' signals promote engulfment).

Caspases prevent ICD during apoptosis, but they are not actually required for the death of the cell. That function is fulfilled by BAK and BAX, two BCL- 2 protein family members. Their activation and subsequent oligomerisation causes mitochondrial outer membrane permeabilization (MOMP), permanently damaging the mitochondria

\footnotetext{
Correspondence: Kate McArthur (kate.mcarthur@monash.edu) or Benjamin T. Kile (benjamin.kile@adelaide.edu.au)

'Department of Biochemistry and Molecular Biology, Biomedicine Discovery Institute, Monash University, Melbourne, VIC 3800, Australia

${ }^{2}$ Faculty of Health and Medical Sciences, The University of Adelaide, Adelaide 5005, Australia

Edited by Ivano Amelio
}

and allowing the release of cytochrome- $c$ into the cytosol. Cytochrome- $c$ is then able to form part of the apoptosome, which sets the caspase cascade into motion. Recently, it has been shown that not only does BAK/BAXmediated MOMP permit the release of mitochondrial inter-membrane space proteins, but also causes extensive remodelling of the mitochondria and exposure of the inner mitochondrial membrane to the cytosol. This phenomenon, called mitochondrial herniation, allows the release of matrix components including mitochondrial DNA (mtDNA) $)^{3,4}$. Cytosolic mtDNA, much like an invading viral genome, is a potent agonist of the cell's innate immune surveillance machinery; the cytosolic DNA sensor Cyclic GMP-AMP Synthase (cGAS) and its signalling counterpart Stimulator of Interferon Genes (STING), which induce rapid Type I IFN secretion ${ }^{5,6}$. Although mtDNA release appears to be a routine event during apoptosis, thanks to rapid caspase-mediated dismantling of the cell, mtDNA-stimulated IFN secretion is only apparent when caspases are disabled.

Apoptotic MOMP has also been shown to trigger autophagy - a process by which cells deliver damaged or potentially toxic cellular components to lysosomes for degradation. Previous reports have shown apoptotic targeting of damaged mitochondria to lysosomes can reduce mtDNA-induced IFN secretion ${ }^{7}$. Thus, apoptotic mtDNA release, when given the opportunity (either by caspase inhibition or autophagy inhibition), is capable of transforming this normally silent cellular fate, into a form of immunogenic cell death (ICD) (Fig. 1).

In this work, Yamazaki and colleagues explore the response of breast cancer cells to radiation therapy (RT) under normal and autophagy-deficient conditions. Using both genetic and pharmacologic inhibition of autophagy, the authors found that autophagy-deficient mouse mammary carcinoma cells were not only more sensitive to RT, 


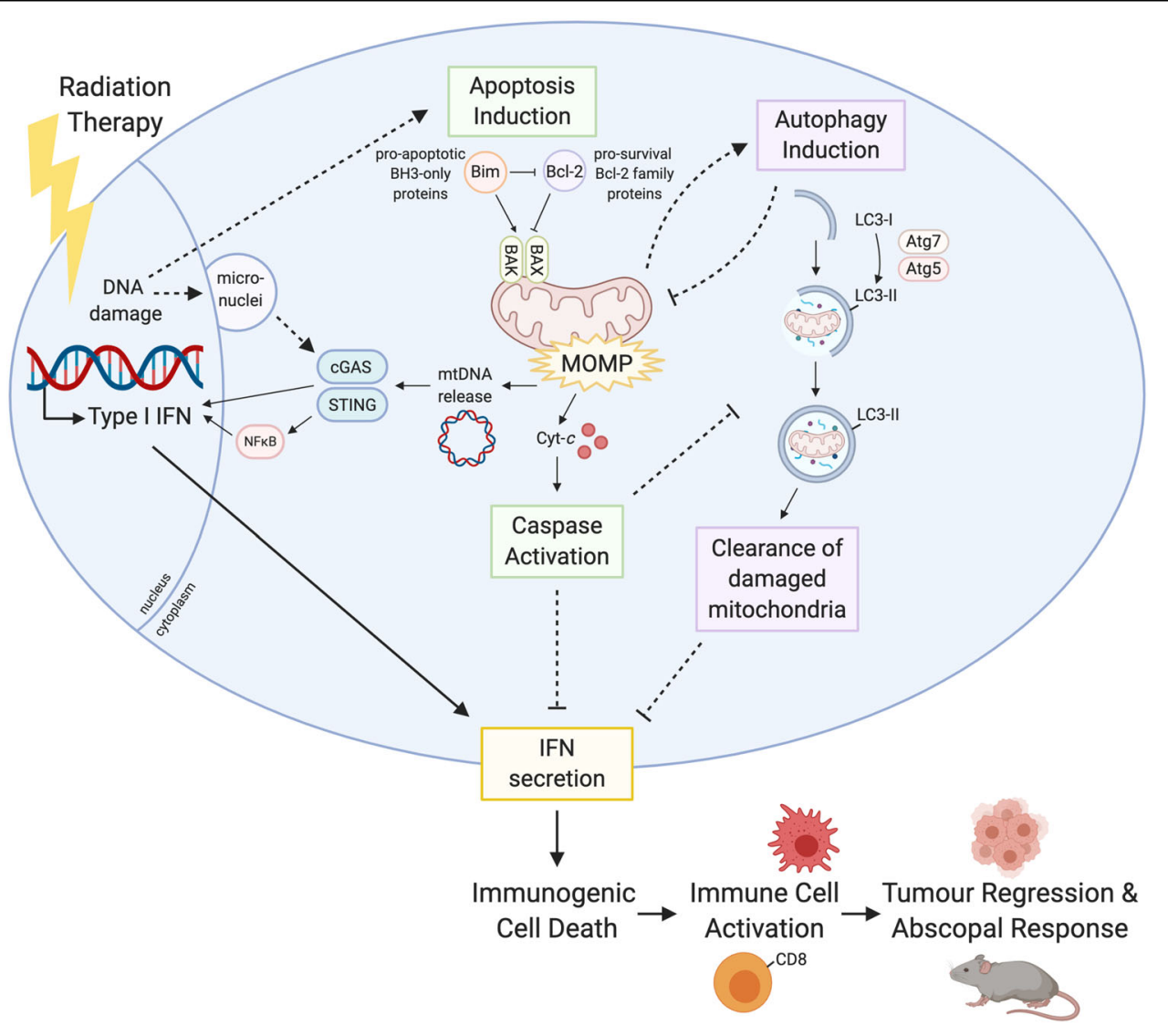

Fig. 1 Summary schematic of the apoptotic and autophagic pathways induced by radiation therapy, and how this leads to immunogenic cell death (ICD). Figure created with BioRender.com.

but produced more IFN in response to it. This was ablated in cGAS-deficient cells.

Previous reports have shown RT can stimulate the formation of micronuclei via DNA damage and genome instability, and that these micronuclei are recognised by cGAS to induce IFN and a pro-inflammatory response ${ }^{8,9}$. These reports also demonstrated that any abscopal responses to RT were also mediated via the cGAS-STING signalling axis. However, these studies differed from this work in one important area: the origin of the DNA recognised by cGAS.

Yamazaki and colleagues employed confocal microscopy to show RT induces the presence of cytosolic DNA foci which are in close proximity to mitochondria, appear to co-localise with mitochondrial transcription factor TFAM (but not with nuclear envelope protein Lamin B) and, most importantly, are absent in cells depleted of mtDNA (termed rho cells): suggesting that in the present model, it is mitochondrial, not genomic, DNA that signals IFN production. The authors demonstrated that RT was unable to induce IFN production in rho cells, and autophagy inhibition did not restore it. Overexpression of anti-apoptotic protein BCL-2, or loss of the pro-apoptotic protein BAX, significantly reduced the presence of cytosolic DNA species and the transcription of IFN following $\mathrm{RT}$. Together, these findings strongly suggest BAK/BAXmediated MOMP and mtDNA release are the drivers of IFN production in response to RT, and that autophagy plays a significant role in attenuating this IFN response in breast cancer cell lines.

Strikingly, when either control or autophagy-deficient cells were injected into the flanks of immunocompetent syngeneic mice (primary tumours), and control cells were injected into the opposing flanks (to model secondary lesions), RT directed at the primary tumours was only able to elicit abscopal responses at non-irradiated sites when the primary tumours were autophagy-deficient. Such responses were further enhanced when combined with CTLA4 inhibition.

These results mirror previous findings from the same group, in which mouse mammary carcinoma cells lacking Caspase-3 were shown to have increased IFN-production in response to RT, which also translated to enhanced abscopal responses (which again were further enhanced 
by CTLA4-blockade) ${ }^{10}$. Thus, despite subtle differences, both autophagy inhibition and caspase inhibition modulate apoptotic IFN production.

In a similar vein, a recent study by Giampazolias et al. ${ }^{11}$, showed that MOMP under caspase-deficient conditions, was able to induce potent anti-tumour effects. In this study, these effects were mtDNA-STING induced, signalled through both IFN-production and NFKB activation $^{11}$. Indeed, there have been a few reports of pharmacological caspase inhibitors combining with cytotoxic treatments to increase anti-tumour responses ${ }^{12,13}$. More recently still, Han et al. ${ }^{14}$, reported Caspase-9 deficient tumours, but not their control-counterparts, demonstrated IFN-dependent CD8 $\mathrm{T}$ cell-mediated complete regression after $\mathrm{RT}$, and impressively, mice remained protected from rechallenge 60 days later ${ }^{14}$.

On the face of it, caspases and autophagy appear to be playing similar roles in the context of apoptosis: shutting down the drivers of ICD, and in particular, mtDNAmediated IFN production. However, several questions remain. First and foremost, why is an autophagic response still critical in caspase-proficient settings? Surely if caspase-mediated proteolysis is rapid and complete, the time-frame within which autophagy would have to clear damaged mitochondria would be too short? Are there ways of inducing MOMP but somehow slowing/decreasing caspase activation, and if so, how is this achieved? Are there differences in the way RT-induced cell death is regulated between different cell/tissue types? As discussed above, there is already evidence that this may be the case, with some groups reporting micronuclei formation and gDNA-cGAS activation, and this study clearly showing MOMP-induced mtDNA-cGAS activation.

A number of pre-clinical trials to establish the ability of autophagy inhibitors or caspase inhibitors to augment anti-cancer therapy are underway (clinicaltrials.gov). However, both drug classes currently come with a number of caveats: the only autophagy inhibitors approved for use are the quinoline derivatives Chloroquine and Hydroxychloroquine, which can have actions beyond autophagy; whilst caspase inhibitors often lack specificity between caspase family members, and generally possess only short half-lives. Encouragingly, more specific autophagy inhibitors (e.g., those targeting ATGB4 and ULK) are beginning to emerge ${ }^{15}$. Perhaps similar advances in caspase-inhibitors will occur. At a conceptual level, there is every reason to believe the approach holds genuine therapeutic potential. Based on the work of Yamazaki and colleagues, and the others discussed here, the prospect of killing dying cells in situ at the same time as manipulating them into driving anti-tumour immunity is very real.

\section{Conflict of interest}

The authors declare that they have no conflict of interest.

\section{Publisher's note}

Springer Nature remains neutral with regard to jurisdictional claims in published maps and institutional affiliations.

Received: 17 August 2020 Revised: 6 September 2020 Accepted: 10 September 2020

Published online: 07 October 2020

\section{References}

1. Yamazaki, T. et al. Mitochondrial DNA drives abscopal responses to radiation that are inhibited by autophagy. Nat. Immunol. 21, 1160-1171 (2020).

2. Kerr, J. F. R., Wyllie, A. H. \& Currie, A. R. Apoptosis: a basic biological phenomenon with wideranging implications in tissue kinetics. Br. J. Cancer 26, 239-257 (1972)

3. McArthur, $\mathrm{K}$. et al. BAK/BAX macropores facilitate mitochondrial herniation and mtDNA efflux during apoptosis. Science 359, eaao6047 (2018).

4. Riley, J. S. et al. Mitochondrial inner membrane permeabilisation enables mtDNA release during apoptosis. EMBO J. 37, e99238 (2018).

5. White, M. J. et al. Apoptotic caspases suppress mtDNA-induced STINGmediated type I IFN production. Cell 159, 1549-1562 (2014).

6. Rongvaux, A. et al. Apoptotic caspases prevent the induction of type I interferons by mitochondrial DNA. Cell 159, 1563-1577 (2014).

7. Lindqvist, L. M. et al. Autophagy induced during apoptosis degrades mitochondria and inhibits type I interferon secretion. Cell Death Differ. 25, 784-796 (2018).

8. Mackenzie, K. J. et al. cGAS surveillance of micronuclei links genome instability to innate immunity. Nature $\mathbf{5 4 8}, \mathbf{4 6 1 - 4 6 5}$ (2017).

9. Harding, S. M. et al. Mitotic progression following DNA damage enables pattern recognition within micronuclei. Nature 548, 466-470 (2017).

10. Rodriguez-Ruiz, M. E. et al. Apoptotic caspases inhibit abscopal responses to radiation and identify a new prognostic biomarker for breast cancer patients. Oncoimmunology 8, e1655964 (2019).

11. Giampazolias, E. et al. Mitochondrial permeabilization engages NF-kappaBdependent anti-tumour activity under caspase deficiency. Nat. Cell Biol. 19, 1116-1129 (2017).

12. Kim, K. W., Moretti, L. \& Lu, B. M867, a novel selective inhibitor of caspase-3 enhances cell death and extends tumor growth delay in irradiated lung cancer models. PLoS ONE 3, e2275 (2008).

13. Moretti, L., Kim, K. W., Jung, D. K., Willey, C. D. \& Lu, B. Radiosensitization of solid tumors by Z-VAD, a pan-caspase inhibitor. Mol. Cancer Therapeut. 8, 1270-1279 (2009).

14. Han, C. et al. Tumor cells suppress radiation-induced immunity by hijacking caspase 9 signaling. Nat. Immunol. 21, 546-554 (2020)

15. Tompkins, K. D. \& Thorburn, A. Focus: death: regulation of apoptosis by autophagy to enhance cancer therapy. Yale J. Biol. Med. 92, 707 (2019). 